\title{
Plasmon assisted photonic crystal quantum dot sensors
}

R. V. Shenoi, D. A. Ramirez, Y. Sharma, R. S. Attaluri, J. Rosenberg, et al.

R. V. Shenoi, D. A. Ramirez, Y. Sharma, R. S. Attaluri, J. Rosenberg, O. J. Painter, S. Krishna, "Plasmon assisted photonic crystal quantum dot sensors," Proc. SPIE 6713, Nanophotonics and Macrophotonics for Space Environments, 67130P (26 September 2007); doi: 10.1117/12.735724

SPIE Event: Optical Engineering + Applications, 2007, San Diego, California, United States 


\title{
Plasmon Assisted Photonic Crystal Quantum Dot Sensors
}

\author{
R. V. Shenoi ${ }^{a}$, D. A. Ramirez ${ }^{a}$, Y. Sharma ${ }^{a}$, R. S. Attaluri ${ }^{a}$, J. Rosenberg ${ }^{b}$, O. J. Painter ${ }^{b}$, \\ and S. Krishna ${ }^{a}$ \\ ${ }^{a}$ Center for High Technology Materials, ECE Department, University of New Mexico, 1313 \\ Goddard St. SE, Albuquerque, New Mexico 87106; \\ ${ }^{b}$ Department of Applied Physics, 1200 East California Boulevard, California Institute of \\ Technology, Pasadena, California 91125.
}

\begin{abstract}
We report Quantum Dot Infrared Detectors (QDIP) where light coupling to the self assembled quantum dots is achieved through plasmons occurring at the metal-semiconductor interface. The detector structure consists of an asymmetric InAs/InGaAs/GaAs dots-in-a-well (DWELL) structure and a thick layer of GaAs sandwiched between two highly doped n-GaAs contact layers, grown on a semi-insulating GaAs substrate. The aperture of the detector is covered with a thin metallic layer which along with the dielectric layer confines light in the vertical direction. Sub-wavelength two-dimensional periodic patterns etched in the metallic layer covering the aperture of the detector and the active region creates a micro-cavity that concentrate light in the active region leading to intersubband transitions between states in the dot and the ones in the well. The sidewalls of the detector were also covered with metal to ensure that there is no leakage of light into the active region other than through the metal covered aperture. An enhanced spectral response when compared to the normal DWELL detector is obtained despite the absence of any aperture in the detector. The spectral response measurements show that the Long Wave InfraRed (LWIR) region is enhanced when compared to the Mid Wave InfraRed (MWIR) region. This may be due to coupling of light into the active region by plasmons that are excited at the metalsemiconductor interface. The patterned metal-dielectric layers act as an optical resonator thereby enhancing the coupling efficiency of light into the active region at the specified frequency. The concept of plasmon-assisted coupling is in principle technology agnostic and can be easily integrated into present day infrared sensors.
\end{abstract}

Keywords: Quantum Dots, Dots-in-a-Well Detector, Photonic Crystal Detector, Plasmons

\section{INTRODUCTION}

Infrared sensors in the 3-25 $\mu \mathrm{m}$ region are highly sought after for applications in missile defense, night vision and fire fighting equipment. QDIP and DWELL detectors have been identified as a promising alternative due to their low dark current and ability to absorb normal incident radiation. It is also possible to leverage the advantage of the mature growth technologies of III-V semiconductors to produce a uniform composition over a large area which is essential for the manufacturing of Focal Planar Arrays (FPA). The current detectors in this regime which are based on Mercury Cadmium Telluride (MCT) do suffer from the spatial nonuniformities in growth which in turn creates a considerable shift in the bandgap. The DWELL detector is a combination of the QDIP and the Quantum Well Infrared Photodetector (QWIP), where the quantum dots are placed inside a quantum well. It makes use of intersubband transitions from the dot to the well and from the dot to the continuum for detection. ${ }^{1}$ The well, when asymmetrically designed offers a bias dependent spectral response that can be used for multi color imaging. The DWELL detector offers several advantages over the QWIP like normal incidence operation, lower dark current and a higher operating temperature. Recently a $640 \times 512$ FPA has been demonstrated using the InAs/InGaAs DWELL detector ${ }^{2}$ and a two color FPA has also been demonstrated using the transition in DWELL detector. As the quantum dots are self assembled during growth ${ }^{3}$ it is not possible to control the size of the dots precisely. Hence the actual emission spectra in the detector occurs from an ensemble of dots each different from the other. This results in broadening of the emission spectrum. However the Quantum Efficiency

Corresponding author: Rajeev V Shenoi

E-mail: rshenoi@ece.unm.edu

Nanophotonics and Macrophotonics for Space Environments, edited by Edward W. Taylor, David A. Cardimona,

Proc. of SPIE Vol. 6713, 67130P, (2007) · 0277-786X/07/\$18 · doi: 10.1117/12.735724 


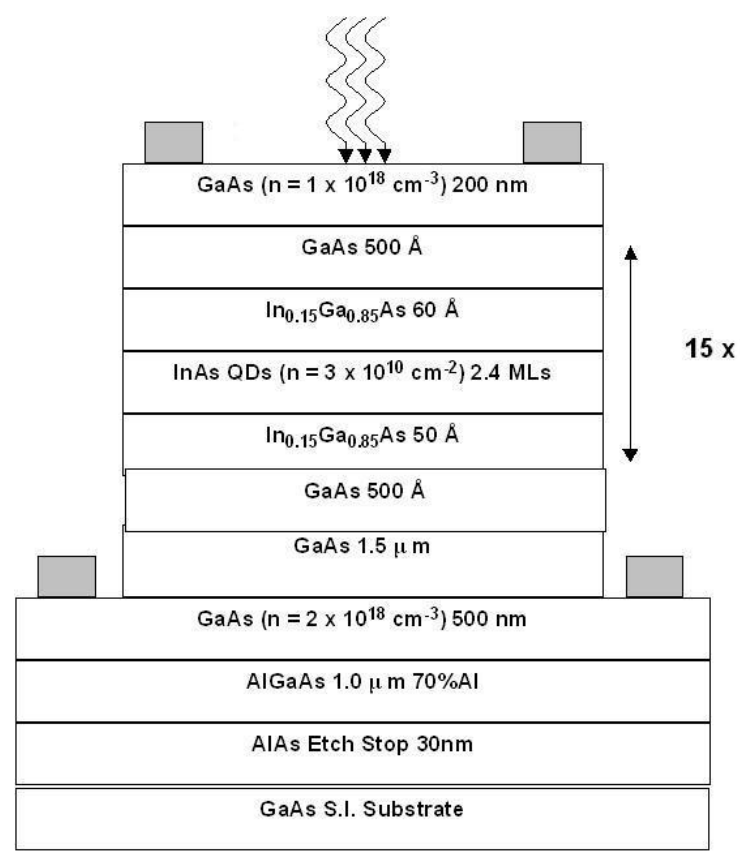

Figure 1. InAs/InGaAs DWELL structure comprising of 15 stacks of active region. The GaAs layer above the bottom contact layer serves as the dielectric waveguide for optical confinement.

(QE) of these systems remain low as the number of absorbing layers possible in a detector structure is limited by the strain needed for the growth of self assembled dots.

The use of a resonant cavity has been suggested to improve the QE and to tailor the spectral characteristics of absorption in quantum dots. The use of a photonic crystal as a resonant cavity has been demonstrated successfully in Refs. 4 and 5. Here we report the fabrication of a DWELL detector where the coupling of light into the active region has been achieved through a photonic crystal pattern etched in a thin plasmonic metal layer on the detector aperture. Here the patterned metal layer is used to couple light into the active region as well as to tailor the absorption spectra. The plasmonic metal layer helps the excitation of surface plasmons at the metal-semiconductor interface that help to guide the light into the active region. The holes are made sufficiently deep so that they facilitate the in-plane waveguiding in the structure. This helps in the greater absorption of light in the active region and improves the responsivity of the detector. This technology is detector agnostic and can easily be adapted for the fabrication of an FPA.

\section{DESIGN AND FABRICATION}

The detectors were grown using solid source Molecular Beam Epitaxy (MBE) as described in Ref.3. As shown in Fig. 1 the active region of the detector consists of 15 layers of asymmetric DWELL structure comprising of 2.4 monolayers of $\mathrm{n}$-doped InAs quantum dots in a $\mathrm{In}_{0.15} \mathrm{Ga}_{0.85}$ As well with GaAs as the barrier. The width of the well below the dot is $5 \mathrm{~nm}$ and above the dot is $6 \mathrm{~nm}$. A $1.5 \mu \mathrm{m}$ thick undoped GaAs layer was grown at the bottom to serve as a dielectric waveguide for confining the optical mode in the active region. A $1 \mu \mathrm{m}$ thick $\mathrm{Al}_{0.7} \mathrm{Ga}_{0.3} \mathrm{As}$ is grown below the bottom contact layer to reduce the coupling of light with the substrate. This is then sandwiched between two highly doped n-GaAs layers that serve as the contact layers. The asymmetry in the well along with the inherent variations in the self assembly of the quantum dots lead to a bias-tunable spectral response for the devices.

The detectors were then processed using standard processing techniques comprising of a mesa etch, passivation and a contact metal deposition to fabricate $400 \times 400 \mu \mathrm{m}^{2}$ pixels with aperture sizes varying from 25 to $300 \mu \mathrm{m}$. A thin layer of metal comprising of $55 \mathrm{~nm}$ Au was then deposited covering the aperture. This serves as the 


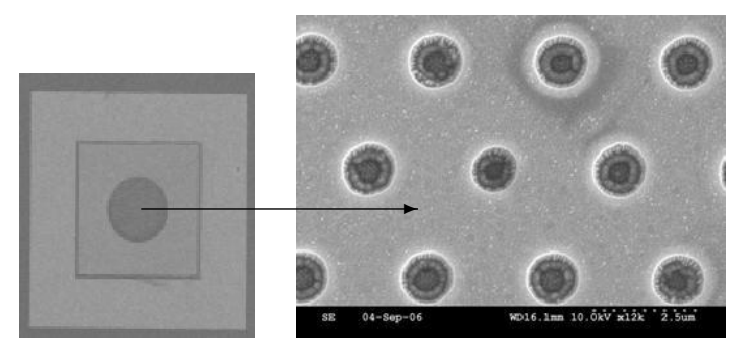

Figure 2. A finished photonic crystal device and the TEM picture of the photonic crystal cavity etched in the top metal layer and the active region.

plasmonic metal layer. The contact metal layer covers the sidewalls of the mesa and prevents the stray coupling of light into the active region.

Hexagonal photonic crystal cavities were then designed and the bandstructure was modeled. The cavity modes are then tuned by varying the radius $r$ and the lattice spacing $a$. The high symmetry points of the photonic crystal, particularly the $\Gamma$ point are targeted as they couple efficiently to normal incidence light. The cavities were designed to obtain resonance at a wavelength of $9 \mu \mathrm{m}$ and the normalized frequency for these structures was obtained to be $a / \lambda=0.348$ which results in a lattice spacing of $3.13 \mu \mathrm{m}$. The patterns were then defined using e-beam lithography and then etched on to the top metal layer and active region. A picture of the finished device and the photonic crystal cavity is shown in Fig.2.

\section{CHARACTERIZATION}

The detectors were characterized through a multi-step process that involved measuring the spectral response, the responsivity, noise power spectral density and the detectivity. Bias dependent spectral response for a pixel with $50 \mu \mathrm{m}$ aperture diameter were measured using a Nicolet 870 Fourier Transform Infrared Spectrometer (FTIR). The devices were measured before the top layer of metal was deposited and after patterning of the top layer metal. The measurement of spectral response after the top layer of metal was deposited everywhere on the device did not yield any results confirming that light is not leaked into the active region by any means. The normalized spectral response of the devices without the plasmonic layer and with the layer at a bias of $2.8 \mathrm{~V}$ are shown in Fig.3. The sample without the plasmonic layer showed spectral peaks centered around 6 and $10 \mu \mathrm{m}$. The short wavelength peak which is due to the transitions from the dot to a higher state in the quantum well, is dominant at lower voltages and the long wavelength peak due to the transitions from the dot to a lower lying state in the well, is pronounced at higher voltages. ${ }^{3}$ This is as result of the extraction of carriers from the lower states in the well due to field assisted tunneling that dominates at higher biases. However the patterned sample shows a marked reduction in the MWIR peak suggesting a strong coupling for the LWIR photon to the active region and a lack of coupling for the MWIR photon.

For characterizing the responsivity and detectivity, the samples were cooled down to liquid nitrogen temperature $(77 \mathrm{~K})$ and were irradiated using a calibrated blackbody at $800 \mathrm{~K}$. The photocurrent was amplified using a SRS 570 low noise amplifier and then measured using a SRS760 Fast Fourier Transform (FFT) spectrum analyzer. The peak responsivity was then computed using the expression

$$
R_{i}=\frac{I_{0}}{\int_{\lambda_{1}}^{\lambda_{2}} \frac{R(\lambda)}{R\left(\lambda_{c}\right)} L_{e}(\lambda, T) A_{s} A_{d} \frac{t F_{F}}{r^{2}} d \lambda}
$$

where $I_{0}, L_{e}, A_{s}, A_{d}$ are the photocurrent, the black body spectral excitance, area of the source, area of the detector and $r, t, F_{F}$ are the the distance between the source and the detector, the transmission of the window and fudge factor respectively. The detectivity $D^{*}$ is then computed as

$$
D^{*}=\frac{\sqrt{A_{d} \Delta f}}{i_{n}} R_{p}
$$




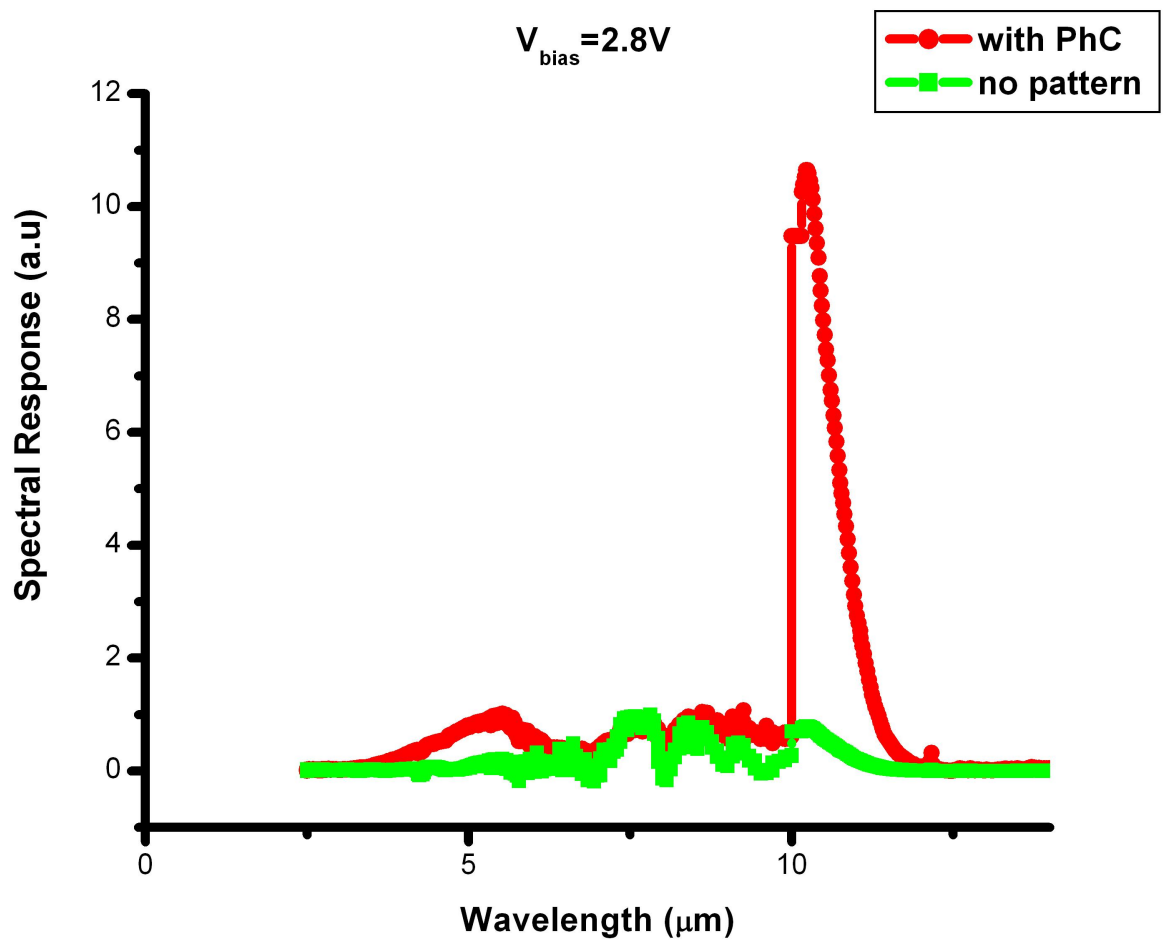

Figure 3. Spectral response of the DWELL detector with and without patterned plasmonic layer at a bias of $2.8 \mathrm{~V}$ at $30 \mathrm{~K}$, normalized with respect to the MWIR peak. The figure shows an enhancement of the LWIR peak in the patterned sample.

where $A_{d}$ is the detector area, $\Delta f$ is the noise equivalent bandwidth, $i_{n}$ is the noise current and $R_{p}$ is the responsivity of the detector. The plots of variation of responsivity and detectivity with the applied bias are shown in Fig.4 and Fig.5

It can be observed that that the responsivity of the patterned sample is higher than that of the sample without plasmonic metal despite the fact that around $40 \%$ of aperture area is removed as result of etching the photonic crystal pattern into the active region. The coupling of light through the top metal layer could be due to the excitation of surface plasmons in the metal semiconductor layer. The change in the spectral characteristics of the samples also support this argument. However the increase in responsivity does not lead to a corresponding increase in the detectivity of the patterned devices. This could be due to the fact that the etch to transfer the photonic crystal pattern might have gone too deep and etched into the bottom contact of the device. This can potentially lead to an increase in the dark current and the noise in the system.

\section{CONCLUSION AND FUTURE WORK}

In this paper we have reported the fabrication of a DWELL detector where the light is coupled to the active region through the excitation of surface plasmons at the metal-semiconductor interface. Various figures of merit of the detector were evaluated and compared to the detector with no patterning. An enhanced responsivity and spectral response have been obtained from the patterned sample. Future work would involve reduction of dark current in the device so as to obtain better detectivity from these devices. The structures need to be optimized for in-plane waveguiding so that the responsivity could be improved further. Tailoring the defects to obtain polarization sensitive detectors or multi-spectral detectors is underway. 


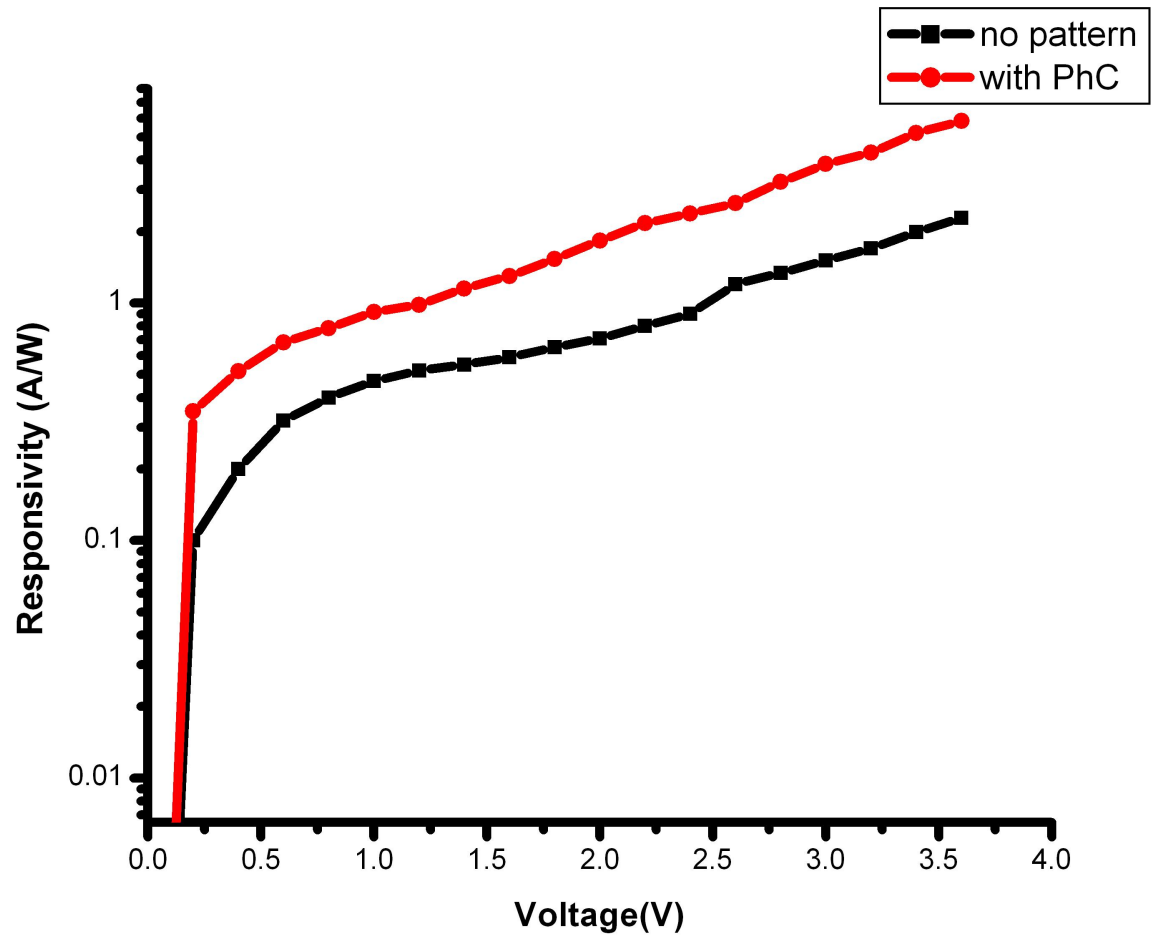

Figure 4. Responsivity of the samples with patterned plasmonic metal and without the plasmonic metal. Measurements performed at $77 \mathrm{~K}$.

\section{ACKNOWLEDGMENTS}

The authors would like to acknowledge support from AFOSR and AFRL for the grant that made this work possible.

\section{REFERENCES}

1. S. Krishna, S. Raghavan, G. von Winckel, A. Stintz, G. Ariyawansa, S. G. Matsik and A. G. U. Perera, Three-color $\left(\lambda_{p 1} \approx 3.8 \mu \mathrm{m}, \lambda_{p 2} \approx 8.5 \mu \mathrm{m}\right.$, and $\left.\lambda_{p 3} \approx 23.2 \mu \mathrm{m}\right)$ InAs/InGaAs quantum-dots-in-a-well detector, Applied Physics Letters, 83, p.p2746, 2003.

2. S. D. Gunapala, S. V. Bandara, C. J. Hill, D. Z. Ting, J. K. Liu; Sir B. Rafol, E. R. Blazejewski, J. M. Mumolo, S. A. Keo, S. Krishna, Y.C. Chang, C. A. Shott, $640 \times 512$ pixels long-wavelength infrared quantum-dot infrared photodetector imaging focal plane array, IEEE Journal of Quantum Electronics, 43, p.p 203, 2007.

3. S. Krishna, Quantum Dots in a Well Detector, Journal of Physics D: Applied Physics, 38, p.p 2147., 2005.

4. K.T. Posani, V. Tripathi, S. Annamalai, N.R. Weisse-Bernstein, Sanjay Krishna, R. Perahia, O. Crisafulli, O.J. Painter, Nanoscale quantum dot infrared sensors with photonic crystal cavity, Applied Physics Letters, $88,2006$.

5. S. Schartner, S. Golka, C. Pflugl, W. Schrenk, A. M. Andrews, T. Roch, and G. Strasser, Band structure mapping of photonic crystal intersubband detectors, Applied Physics Letters, 89, 2006. 


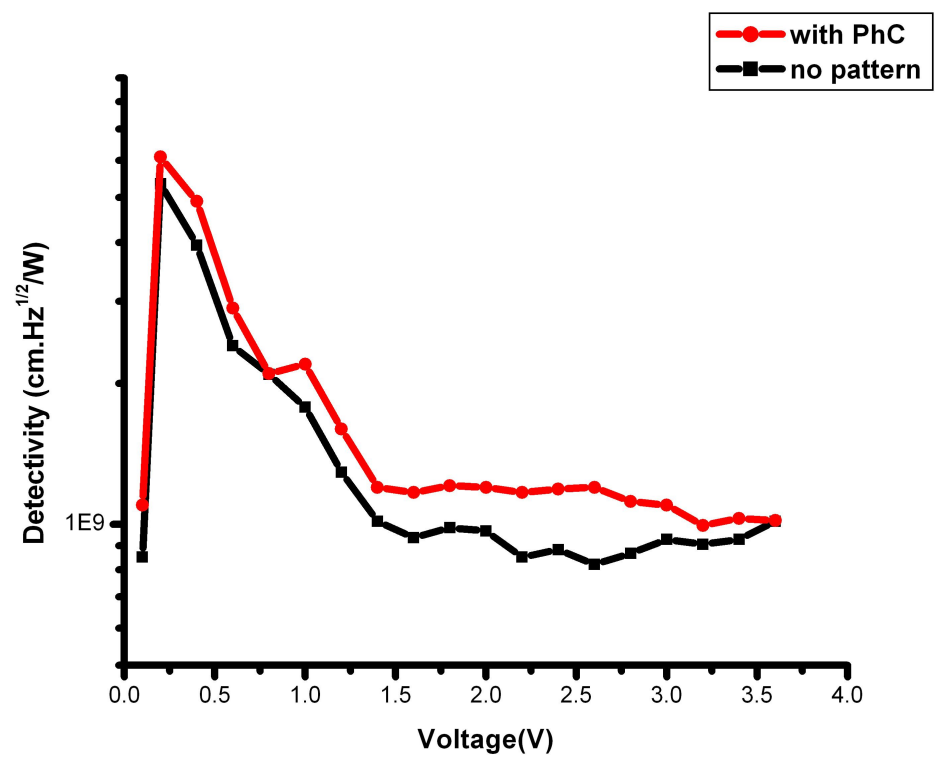

Figure 5. Detectivity of the samples with patterned plasmonic metal and without the plasmonic metal. 